\title{
HOOK YOUNG DIAGRAMS, COMBINATORICS AND REPRESENTATIONS OF LIE SUPERALGEBRAS
}

\section{A. BERELE ${ }^{1}$ AND A. REGEV ${ }^{2}$}

Let $V$ be a finite-dimensional $F$-vector space, $\operatorname{char}(F)=0$. Schur introduced the action of the symmetric group $S_{n}$ on $V^{\otimes n}$ and was then able to determine the representation theory of the general linear group $\mathrm{GL}(V)[9]$. His work was later completed by $\mathrm{H}$. Weyl [10]. This work connects that representation theory with combinatorics via standard and semistandard Young tableaux and via the Schur functions (cf. [6]). Many of the objects in this theory are parametrized by the Young diagrams in a strip.

In this work we introduce a slightly more general permutation action of $S_{n}$ on $V^{\otimes n}$ and then describe how most of the above theory generalizes. The main feature here is that most of the generalized objects are parametrized by the partitions inside a hook.

The action. Let $k, l \geq 0, k+l>0, T$ and $U$ disjoint vector spaces, $\operatorname{dim} T=$ $k, \operatorname{dim} U=l$, and $V=T \oplus U$. We define a new right action of $S_{n}$ on $V^{\otimes n}$, i.e., a map $\psi: S_{n} \rightarrow \operatorname{End}_{F}\left(V^{\otimes n}\right)$, based on Schur's original action and on the functions $f_{I}: S_{n} \rightarrow\{ \pm 1\}[5]$ as follows. Choose bases $t_{1}, \ldots, t_{k} \in T$, $u_{1}, \ldots, u_{l} \in U$. These induce a basis of $V^{\otimes n}$. Let $v_{1} \otimes \cdots \otimes v_{n} \in V^{\otimes n}$, $v_{1}, \ldots, v_{n} \in\left\{t_{1}, \ldots, u_{l}\right\}$ be such a basis element, let $I=\left\{i \mid v_{i} \in U\right\}$ and let $\sigma \in S_{n}$. Then

$$
\left(v_{1} \otimes \cdots \otimes v_{n}\right) \psi(\sigma)_{\mathrm{DEF}}^{=} f_{I}(\sigma)\left(v_{\sigma(1)} \otimes \cdots \otimes v_{\sigma(n)}\right) .
$$

Extend $\psi(\sigma)$ to all of $V^{\otimes n}$ by linearity: $\psi(\sigma) \in \operatorname{End}\left(V^{\otimes n}\right)$. As usual, we now extend $\psi$ to $F S_{n}$, then check that $\psi: F S_{n} \rightarrow \operatorname{End}\left(V^{\otimes n}\right)$ is an (associative) algebra homomorphism.

The question. It is well known that $F S_{n}=\sum_{\lambda \in \operatorname{Par}(n)} \oplus I_{\lambda}$, where $\operatorname{Par}(n)$ denotes the set of partitions of $n$ and where each $I_{\lambda}$ is a simple algebra. It follows that for some $\Gamma=\Gamma(k, l ; n) \subseteq \operatorname{Par}(n), \psi\left(F S_{n}\right) \cong \sum_{\lambda \in \Gamma} \oplus I_{\lambda}$, and the basic question here is to describe $\Gamma$. Letting $B(k, l ; n)$ be the centralizer of $\psi\left(F S_{n}\right)$ in $\operatorname{End}_{F}\left(V^{\otimes n}\right)$, the decomposition of $V^{\otimes n}$ into irreducible $\psi\left(F S_{n}\right)$ or $B(k, l ; n)$ modules, will be given by the classical theory of Schur.

The answer, which extends a theorem of Weyl is

THE HOOK THEOREM. Let

$$
H(k, l ; n)=\left\{\lambda=\left(\lambda_{1}, \lambda_{2}, \ldots\right) \in \operatorname{Par}(n) \mid \lambda_{j} \leq l \text { if } j \geq k+1\right\}
$$

Received by the editors April 26, 1982.

1980 Mathematics Subject Classification. Primary 05A15, 05A17, 17A70, 17B10, 20C30; Secondary 16A38, 81C40.

${ }^{1}$ Supported by a grant from the Bantrell Foundation.

${ }^{2}$ Partially supported by grant $\mathrm{M}(\mathrm{T})$ from the Israel Academy of Sciences \& Humanities. 
(i.e., $\lambda$ is included in the hook

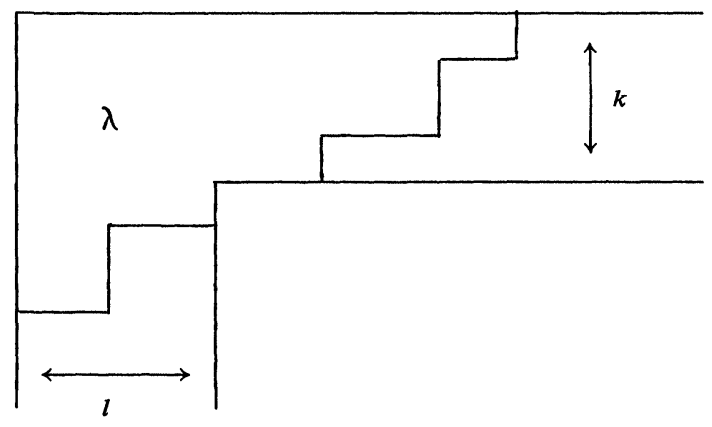

Then $\Gamma=H(k, l ; n)$.

One proof of that theorem is by Young's (or the Littlewood-Richardson) rule.

A second proof is a slight generalization of a proof, based on a dimension argument, of the corresponding theorem of $\mathrm{H}$. Weyl. It requires the notion of $(k, l)$ semistandard tableaux: The tableau $T_{\lambda}$ (of shape $\lambda, \lambda \in \operatorname{Par}(n)$ ) is $(k, l)$ semistandard if there is a partition $\mu, \mu<\lambda$, such that the $\mu$ subtableau of $T_{\lambda}$ is semistandard on $t_{1}, \ldots, t_{k}$ and the conjugate of the skew tableau $\left(T_{\lambda / \mu}\right)$ is semistandard on $u_{1}, \ldots, u_{l}$. The number of such tableaux of shape $\lambda$ is denoted $s_{k, l}(\lambda)$. (We completely determine these numbers.) We now generalize the Robinson-Schensted correspondence [4] to two sets of variables (there are many such generalizations) and prove

THEOREM. The generalized correspondence induces a bijection between the set of basis elements of $V^{\otimes n}$ and the set of all pairs of tableaux $(P, Q)$ where $P$ is $(k, l)$ semistandard and $Q$ is standard, both of the same shape $\lambda, \lambda \in \operatorname{Par}(n)$.

These are the main ingredients of that second proof. They are closely related to the representation theory of the General Linear Lie superalgebra $p l(V),[8, \mathbf{2}, \mathbf{3}]$.

Representations of $p l(V)$. Let $V=T \oplus U$ as before, call $V_{\overline{0}}=T, V_{\overline{1}}=U$, and let $p l(V)$ be the corresponding General Linear Lie superalgebra [8]. We define a Lie superhomomorphism $g: p l(V) \rightarrow \operatorname{End}\left(V^{\otimes n}\right)$ by sending a matrix to the corresponding superderivation and prove

THEOREM. The associative algebra generated by $g(p l(V))$ equals $B(k, l ; n)$.

In particular, we get a complete decomposition of $V^{\otimes n}$ into $p l(V)$ irreducible modules (from the $B(k, l ; n)$ decomposition). Thus, for each $n$ we obtain very explicit $p l(V)$ irreducible representations parametrized by $\lambda \in$ $H(k, l ; n)$ and of dimensions $s_{k, l}(\lambda)$.

Note that a comprehensive (finite-dimensional) representation theory for the classical Lie superalgebras has been worked out, essentially via the highest weight approach $[2,3]$. 
Some applications. The explicitness of our representations allows us to prove branching rules for them, which generalize the branching rule for the representations of $\mathrm{GL}(k)$.

Many combinatorial objects that occur in the above theory are "hook analogues" of classical objects, like the Schur functions, Kostka numbers and others.

These results also have many applications to the theory of P.I. algebras because of the "hook property" of the multilinear cocharacters of such algebras [1]. Because of this property the classical theory does not seem to suffice for these applications [7].

The details of all this will be published elsewhere.

\section{REFERENCES}

1. S. A. Amitsur and A. Regev, P.I.-algebras and their cocharacters (preprint).

2. V. G. Kac, Lie superalgebras, Adv. in Math. 26 (1977), 8-96.

3. _ Characters of typical representations of classical Lie superalgebras, Comm. Algebra 5 (1977), 889-897.

4. D. E. Knuth, The art of computer programming, vol. 3, Addison-Wesley, Reading, Mass., 1968.

5. D. Krakowski and A. Regev, The polynomial identities of the Grassmann algebra, Trans. Amer. Math. Soc. 181 (1973), 429-438.

6. I. G. Macdonald, Symmetric functions and Hall polynomials, Clarendon Press, Oxford, 1979.

7. A. Regev, The Kronecker product of $S_{n}$-characters and an $A \otimes B$ theorem for Capelli identities, J. Algebra 66 (1980), 505-510.

8. M. Scheunert, The theory of Lie superalgebras, Lecture Notes in Math., vol. 716, SpringerVerlag, Berlin and New York, 1979.

9. I. Schur, Ueber eine Klasse von Matrizen, die sich einer gegebenen Matrix zuordnen lassen, Dissertation, Berlin, 1901.

10. H. Weyl, The classical groups, Princeton Math. Series No. 1, Princeton, N.J., 1939.

Department of Theoretical Mathematics, The Weizmann Institute OF SCIENCE, REHOVOT 76100, ISRAEL

Current address: (A. Berele) Department of Mathematics, University of California at San Diego, La Jolla, California 92093

Current address: (A. Regev) Department of Mathematics, Brandeis University, Waltham, Massachusetts 02154 